\title{
On gravitational radiation and the energy flux of matter
}

\author{
J. W. Maluf* and F. F. Faria \\ Instituto de Física, \\ Universidade de Brasília \\ C. P. 04385 \\ 70.919-970 Brasília DF, Brazil
}

\begin{abstract}
A suitable derivative of Einstein's equations in the framework of the teleparallel equivalent of general relativity (TEGR) yields a continuity equation for the gravitational energy-momentum. In particular, the time derivative of the total gravitational energy is given by the sum of the total fluxes of gravitational and matter fields energy. We carry out a detalied analysis of the continuity equation in the context of Bondi and Vaidya's metrics. In the former space-time the flux of gravitational energy is given by the well known expression in terms of the square of the news function. It is known that the energy definition in the realm of the TEGR yields the ADM (Arnowitt-Deser-Misner) energy for appropriate boundary conditions. Here we show that the same energy definition also describes the Bondi energy. The analysis of the continuity equation in Vaidya's space-time shows that the variation of the total gravitational energy is determined by the energy flux of matter only.
\end{abstract}

PACS numbers: 04.20.Cv, 04.20.Fy

(*) e-mail: wadih@fis.unb.br 


\section{Introduction}

In spite of its wide acceptance as a theory for the dynamics of the spacetime and of the gravitational field, Einstein's theory of general relativity, in the metrical formulation, does not allow the emergence of an unequivocal definition of gravitational energy. The definition of such quantity is surely important for a comprehensive understanding of the theory. In the past the major attempts at defining the gravitational energy were carried out by means of pseudotensors $[1,2,3]$. Nowadays the concept of quasilocal energy (i.e., the energy associated to a closed spacelike 2 -surface) is being thoroughly investigated in the context of Hamiltonian formulations (see, for instance, Ref. [4] and references therein). Although this approach is promising, there is no ultimate consensus about it.

Two well established notions of gravitational energy are the total energy of the space-time, known as the ADM (Arnowitt-Deser-Misner) energy [5], evaluated on a spacelike slice of a space-time that is asymptotically flat, and the Bondi energy [6] that describes the mass of radiating systems in asymptotically flat space-times. The latter is usually defined at null infinity. A remarkable feature of the Bondi energy is that it is related (by means of the field equations) to the loss of the total mass of a source that radiates gravitational waves. The loss of total mass is given in terms of minus the square of the news function, and therefore the mass of the source can only decrease.

A definition for the gravitational energy-momentum has been investigated in the realm of the teleparallel equivalent of general relativity (TEGR) $[7,8]$ (Ref. [9] considers the TEGR as a particular case of metric-affine theories of gravity, and provides a large number of references on the subject). Attempts to define the gravitational energy in the context of teleparallel theories of gravity were first considered by Møller [10], who noticed that an expression for the gravitational energy density could be constructed out of the torsion tensor. Arguments in favour of the localizability of the gravitational energy are presented in Refs. [7, 8, 11], where it is argued that it is possible to identify the gravitational energy density with an appropriate scalar density, defined in terms of a set of global tetrad fields, which is a total divergence. Therefore the gravitational energy density in consideration transforms as a scalar density under coordinate transformations. We recall that the local- 
izability of the gravitational energy was already supported by Bondi [12], who analyzed the possible forms of energy transfer of the gravitational field. He claimed that in relativity a nonlocalizable form of energy is inadmissible, because all forms of energy contribute to gravity, and therefore its location can in principle be found.

However, it is reasonable to expect the localization of the gravitational energy to depend on the reference frame [11]. The Principle of Equivalence is based on the equality of inertial and gravitational masses. Alternatively, an accelerated frame can be locally considered as a rest frame with the addition of a certain gravitational field. Therefere our perception of the strength of the gravitational field on nearby bodies depends clearly on our reference frame, and so does the gravitational energy as measured on the same frame. Reference frames are described in terms of tetrad fields [13, 14], which are the basic field quantities of the TEGR. The total energy of a space-time, however, is not expected to depend on a particular frame (the total gravitational energy of any asymptotically flat space-time, in the TEGR, is precisely the ADM energy [7]). The dependence of the localization of the gravitational energy on the frame is a manifestation of the fact that gravitational and inertial forces are of the same nature.

In this article we address the issue of the variation of the gravitational energy and the corresponding total flux of energy at spacelike infinity. The success of the present analysis may qualify the TEGR as an adequate framework to investigate more intricate astrophysical models of energy flux and gravitational waves emission. We conclude that the energy-momentum definition in consideration here provides a unique framework to analyze both the ADM and the Bondi energy. We will show that for the Bondi space-time metric tensor the decrease in time of the total gravitational energy causes the flux of gravitational energy only (by means of the emission of gravitational waves), whereas in Vaidya's space-time $[15,16]$ the loss of gravitational energy is related to a pure flux of matter energy. The variation in time of the gravitational energy is determined by the sum of the gravitational and matter energy fluxes. From the point of view of Bondi [12], these fluxes characterize the intangible and tangible forms of gravitational energy transfer, respectively (the latter being determined by the energy-momentum tensor).

In section II of the article we recall the simple derivation of the continuity equation for the gravitational energy-momentum. In section III we present the construction of the simplest set of tetrad fields, defined in terms of the 
retarded time $u=t-r$, that describes the Bondi space-time. The Bondi energy, the loss of total mass and the total flux of gravitational energy at spacelike infinity are obtained in section IV. In section $\mathrm{V}$ we consider the Vaidya space-time metric tensor and relate the loss of the total mass of the source to a pure matter energy flux. Finally in section VI we present our conclusions.

Notation: space-time indices $\mu, \nu, \ldots$ and $\mathrm{SO}(3,1)$ indices $a, b, \ldots$ run from 0 to 3. Time and space indices are indicated according to $\mu=0, i, \quad a=(0),(i)$. The tetrad field $e^{a}{ }_{\mu}$ yields the definition of the torsion tensor: $T^{a}{ }_{\mu \nu}=$ $\partial_{\mu} e^{a}{ }_{\nu}-\partial_{\nu} e^{a}{ }_{\mu}$. The flat, Minkowski space-time metric is fixed by $\eta_{a b}=$ $e_{a \mu} e_{b \nu} g^{\mu \nu}=(-+++)$.

\section{The fluxes of gravitational and matter fields energy}

The Lagrangian density for the graviational field in the TEGR, in the presence of matter fields, is given by

$$
\begin{aligned}
L\left(e_{a \mu}\right) & =-k e\left(\frac{1}{4} T^{a b c} T_{a b c}+\frac{1}{2} T^{a b c} T_{b a c}-T^{a} T_{a}\right)-L_{m} \\
& \equiv-k e \sum^{a b c} T_{a b c}-L_{m},
\end{aligned}
$$

where $k=1 /(16 \pi)$ and $e=\operatorname{det}\left(e^{a}{ }_{\mu}\right)$. The tensor $\Sigma^{a b c}$ is defined by

$$
\Sigma^{a b c}=\frac{1}{4}\left(T^{a b c}+T^{b a c}-T^{c a b}\right)+\frac{1}{2}\left(\eta^{a c} T^{b}-\eta^{a b} T^{c}\right),
$$

and $T^{a}=T_{b}^{b}{ }^{a}$. The quadratic combination $\Sigma^{a b c} T_{a b c}$ is proportional to the scalar curvature $R(e)$, except for a total divergence [17]. $L_{m}$ represents the Lagrangian density for matter fields. The field equations for the tetrad field read

$$
e_{a \lambda} e_{b \mu} \partial_{\nu}\left(e \Sigma^{b \lambda \nu}\right)-e\left(\Sigma^{b \nu}{ }_{a} T_{b \nu \mu}-\frac{1}{4} e_{a \mu} T_{b c d} \Sigma^{b c d}\right)=\frac{1}{4 k} e T_{a \mu}
$$

where 


$$
\frac{\delta L_{m}}{\delta e^{a \mu}} \equiv e T_{a \mu}
$$

It is possible to prove by explicit calculations that the left hand side of Eq. (3) is exactly given by $\frac{1}{2} e\left[R_{a \mu}(e)-\frac{1}{2} e_{a \mu} R(e)\right]$.

Multiplication of Eq. (3) by the appropriate inverse tetrad fields yields the latter equation in the form

$$
\partial_{\nu}\left(-4 k e \Sigma^{a \lambda \nu}\right)=-k e e^{a \mu}\left(4 \Sigma^{b \lambda \nu} T_{b \nu \mu}-\delta_{\mu}^{\lambda} \Sigma^{b d c} T_{b c d}\right)-e e_{\mu}^{a} T^{\lambda \mu} .
$$

As usual, tetrad fields convert space-time into Lorentz indices and vice-versa. By making $\lambda=j$ (i.e., by restricting the space-time index $\lambda$ to assume only space values) we obtain

$$
\begin{aligned}
-\partial_{0}\left(-4 k e \Sigma^{a 0 j}\right)-\partial_{k}\left(-4 k e \Sigma^{a k j}\right)= & -k e e^{a \mu}\left(4 \Sigma^{b c j} T_{b c \mu}-\delta_{\mu}^{j} \Sigma^{b c d} T_{b c d}\right) \\
& -e e^{a}{ }_{\mu} T^{j \mu} .
\end{aligned}
$$

Now we take the derivative of the equation above with respect to $j$. The second term on the left hand side vanishes because of the antisymmetry property $\Sigma^{a k j}=-\Sigma^{a j k}$. The resulting equation reads

$$
\begin{aligned}
-\partial_{0} \partial_{j}\left(-4 k e \Sigma^{a 0 j}\right)= & -k \partial_{j}\left[e e^{a \mu}\left(4 \Sigma^{b c j} T_{b c \mu}-\delta_{\mu}^{j} \Sigma^{b c d} T_{b c d}\right)\right] \\
& -\partial_{j}\left(e e_{\mu}^{a} T^{j \mu}\right) .
\end{aligned}
$$

We recall that in the Hamiltonian formulation of the TEGR [18] the momentum canonically conjugated to the tetrad components $e_{a j}$ is given by $\Pi^{a j}=-4 k e \sum^{a 0 j}$, and that the gravitational energy-momentum $P^{a}$ contained within a volume $V$ of the three-dimensional spacelike hypersurface is defined by $[7]$

$$
P^{a}=-\int_{V} d^{3} x \partial_{j} \Pi^{a j}
$$

If no condition is imposed on the tetrad fields, $P^{a}$ transforms as a vector under the global $\mathrm{SO}(3,1)$ group. (The Hamiltonian formulation of the TEGR has also been extensively discussed by Blagojević and Nikolić [19].) 
By integrating Eq. (6) on a volume $V$ of the three-dimensional space we arrive at

$$
\frac{d}{d t}\left[-\int_{V} d^{3} x \partial_{j} \Pi^{a j}\right]=-\Phi_{g}^{a}-\Phi_{m}^{a}
$$

where

$$
\Phi_{g}^{a}=k \int_{S} d S_{j}\left[e e^{a \mu}\left(4 \Sigma^{b c j} T_{b c \mu}-\delta_{\mu}^{j} \Sigma^{b c d} T_{b c d}\right)\right],
$$

is a component of the gravitational energy-momentum flux, and

$$
\Phi_{m}^{a}=\int_{S} d S_{j}\left(e e_{\mu}^{a} T^{j \mu}\right),
$$

is the $a$ component of the matter energy-momentum flux. $S$ represents the spatial boundary of the volume $V$. Therefore the loss of gravitational energy $P^{(0)}=E$ is governed by the equation

$$
\frac{d E}{d t}=-\Phi_{g}^{(0)}-\Phi_{m}^{(0)}
$$

Equation (8) has been derived previously in the Lagrangian formulation in Ref. [11], without the addition of matter fields. It does not depend on details of any Hamiltonian formulation. The time variable $t$ may also stand for the retarded time $u$. We note that in the analysis of the Hamiltonian formulation of the gravitational field in null surfaces, in the framework of the TEGR [20], the energy definition given by Eq. (7) was already anticipated. However the investigation of the gravitational energy for the Bondi space-time metric tensor was first carried out by imposing the time gauge condition [21] (use was not made of the retarded time), a framework that is not quite suitable for such analysis. In the following sections we will show that $\Phi_{g}^{(0)}$ plays a major role to Bondi's radiating space-time, since it yields the well known

expression for the gravitational energy loss, whereas only $\Phi_{m}^{(0)}$ is relevant to Vaidya's space-time.

\section{The tetrad field for the Bondi space-time}

The tetrad field for the flat space-time, in cartesian coordinates, is given by $e^{a}{ }_{\mu}(c t, x, y, z)=\delta_{\mu}^{a}$. By making a coordinate transformation $t \rightarrow u$, where 
$u=t-r / c$ is the retarded time and $r=\sqrt{x^{2}+y^{2}+z^{2}}$, we obtain

$$
e_{\mu}^{a}(u, x, y, z)=\left(\begin{array}{cccc}
1 & x / r c & y / r c & z / r c \\
0 & 1 & 0 & 0 \\
0 & 0 & 1 & 0 \\
0 & 0 & 0 & 1
\end{array}\right)
$$

where $a$ and $\mu$ label the line and column indices, respectively. In spherical coordinates we have

$$
e_{\mu}^{a}(u, r, \theta, \phi)=\left(\begin{array}{cccc}
1 & 1 / c & 0 & 0 \\
0 & \sin \theta \cos \phi & r \cos \theta \cos \phi & -r \sin \theta \sin \phi \\
0 & \sin \theta \sin \phi & r \cos \theta \sin \phi & r \sin \theta \cos \phi \\
0 & \cos \theta & -r \sin \theta & 0
\end{array}\right) .
$$

In Eqs. (12) and (13) only $c$ stands for the velocity of light.

The set of tetrad fields given by Eq. (12) defines a holonomic transformation $d q^{a}=e^{a}{ }_{\mu} d x^{\mu}$ between the coordinates $q^{(0)}=u+r / c, q^{(1)}=x, q^{(2)}=y$ and $q^{(3)}=z$ that describe a reference space-time [7], and the space-time coordinates $(u, x, y, z)$. In the form given by Eq. (12) the tetrad fields violate the time gauge condition because $e_{(0) i} \neq 0$, and therefore $e_{(i)}{ }^{0} \neq 0$. A nontrivial manifestation of the gravitational field takes place when the transformation $d q^{a}=e^{a}{ }_{\mu} d x^{\mu}$ becomes anholonomic [7].

It is known that the use of the retarded time $u$ clearly simplifies the description of the Bondi space-time, since in this case the null surface condition $g^{00}=0$ can be easily achieved. However it is not possible to impose at the same time the condition $g^{00}=0$ and the time gauge condition.

In the flat space-time the violation of the time gauge condition may be associated to a boost of the physical space-time with coordinates $x^{\mu}$ with respect to the reference space-time with coordinates $q^{a}[7]$. The boost singles out a particular direction in space. If the time gauge condition is to be violated in the description of a radiating gravitational field, then it should not privilege any direction in space, and ideally the violation should be isotropic. This feature is manifest in the flat space-time tetrad field given by Eq. (12), and it will characterize the simplest set of tetrad fields that describes the Bondi space-time metric tensor, as we will see.

The Bondi metric tensor is given by 


$$
\begin{aligned}
d s^{2}= & -\left(\frac{V}{r} e^{2 \beta}-U^{2} r^{2} e^{2 \gamma}\right) d u^{2}-2 e^{2 \beta} d u d r \\
& -2 U r^{2} e^{2 \gamma} d u d \theta+r^{2}\left(e^{2 \gamma} d \theta^{2}+e^{-2 \gamma} \sin ^{2} \theta d \phi^{2}\right),
\end{aligned}
$$

where $(r, \theta, \phi)$ are the usual spherical coordinates and $u$ is the retarded time. The functions in the equation above have the following asymptotic behaviour

$$
\begin{aligned}
\beta= & -\frac{c^{2}}{4 r^{2}}+\cdots \\
\gamma= & \frac{c}{r}+\cdots \\
\frac{V}{r}= & 1-\frac{2 M}{r} \\
& -\frac{1}{r^{2}}\left[\frac{\partial d}{\partial \theta}+d \cos \theta-\left(\frac{\partial c}{\partial \theta}\right)^{2}-4 c\left(\frac{\partial c}{\partial \theta}\right) \cot \theta\right. \\
& \left.-\frac{1}{2} c^{2}\left(1+8 \cot ^{2} \theta\right)\right]+\cdots \\
U= & -\frac{1}{r^{2}}\left(\frac{\partial c}{\partial \theta}+2 c \cot \theta\right) \\
& +\frac{1}{r^{3}}\left(2 d+3 c \frac{\partial c}{\partial \theta} \cot \theta+4 c^{2} \cot \theta\right)+\cdots,
\end{aligned}
$$

where $M=M(u, \theta)$ and $d=d(u, \theta)$ are the mass aspect and the dipole aspect, respectively, and from the function $c(u, \theta)$ we define the news function $\dot{c}=(\partial c(u, \theta)) / \partial u$. The inverse metric tensor is given by

$$
g^{\mu \nu}(u, r, \theta, \phi)=\left(\begin{array}{cccc}
0 & -e^{-2 \beta} & 0 & 0 \\
-e^{-2 \beta} & e^{-2 \beta} \frac{V}{r} & -e^{-2 \beta} U & 0 \\
0 & -e^{-2 \beta} U & \frac{e^{-2 \gamma}}{r^{2}} & 0 \\
0 & 0 & 0 & \frac{e^{2 \gamma}}{r^{2} \sin ^{2} \theta}
\end{array}\right),
$$

and the determinant of $e^{a}{ }_{\mu}$ reads $e=\sqrt{-g}=e^{2 \beta} r^{2} \sin ^{2} \theta$.

The simplest set of tetrad fields $e_{a \mu}(u, r, \theta, \phi)$ that leads to Eq. (14) is precisely the one for which the violation of the time gauge condition is isotropic. It reads 


$$
e_{a \mu}=\left(\begin{array}{cccc}
-A & -B & 0 & 0 \\
C \cos \theta \cos \phi & B \sin \theta \cos \phi & r e^{\gamma} \cos \theta \cos \phi & -r e^{-\gamma} \sin \theta \sin \phi \\
C \cos \theta \sin \phi & B \sin \theta \sin \phi & r e^{\gamma} \cos \theta \sin \phi & r e^{-\gamma} \sin \theta \cos \phi \\
-C \sin \theta & B \cos \theta & -r e^{\gamma} \sin \theta & 0
\end{array}\right)
$$

The functions $A, B$ and $C$ are defined by

$$
\begin{aligned}
& A=e^{\beta}\left(\frac{V}{r}\right)^{\frac{1}{2}}, \\
& B=e^{\beta}\left(\frac{V}{r}\right)^{-\frac{1}{2}}, \\
& C=-U r e^{\gamma} .
\end{aligned}
$$

It is possible to show by means of a coordinate transformation that

$$
\begin{aligned}
e^{(0)}{ }_{1}(u, x, y, z) & =\frac{x}{r} e^{\beta}\left(\frac{V}{r}\right)^{-\frac{1}{2}} \\
e^{(0)}{ }_{2}(u, x, y, z) & =\frac{y}{r} e^{\beta}\left(\frac{V}{r}\right)^{-\frac{1}{2}} \\
e^{(0)}{ }_{3}(u, x, y, z) & =\frac{z}{r} e^{\beta}\left(\frac{V}{r}\right)^{-\frac{1}{2}} .
\end{aligned}
$$

Therefore the feature above mentioned regarding the violation of the time gauge condition is manifest in Eq. (19) (of course the functions $\beta$ and $V$ depend on $\theta$ ). In this respect Eq. (19) shares a similarity with Eq. (12). Moreover the spatial components of $e_{a \mu}$ in Eq. (17), in cartesian coordinates, satisfy

$$
e_{(i) j}(u, x, y, z)=e_{(j) i}(u, x, y, z) .
$$

For the flat space-time tetrad field Eq. (20) ensures that the physical spacetime with coordinates $x^{\mu}$ is not rotating with respect to the reference spacetime with coordinates $q^{a}$. This condition is required to hold for a set of tetrad fields that describe an arbitrary gravitational field [7]. 
The set of tetrad fields given by Eq. (17) is fixed precisely by the six conditions determined by Eqs. (19) and (20). We propose that the isotropic violation of the time gauge condition, given in the present context by Eq. (19), together with the condition defined by Eq. (20) fix the structure of any set of tetrad fields for radiating space-time metric tensors determined in terms of the retarded time $u$, in which case the imposition of the time gauge condition is troublesome. We argue that such tetrad field structure is adapted to static observers at spacelike infinity (because of Eq. (20)) and is suitable to investigate the properties of the energy flux of a system that emits gravitational waves, as we will see.

Before closing this section we note that the idea of a preferred frame has been recently considered in connection with investigations either in cosmology or in attempts to the quantum theory of gravity [22]. The motivation behind such attempts to a preferred frame are quite different from our purposes, and even the approach itself is conceptually and mathematically different from the what has been presented above. Nevertheless it is worth noting that similar ideas emerge in several distinct analyses in general relativity.

\section{Bondi energy and the energy loss}

By considering $a=(0)$ in Eq. 7 we have an expression for the energy of the gravitational field contained within a volume $V$ of the three-dimensional spacelike hypersurface, bounded by a surface $S$ of constant radius $r$,

$$
\begin{aligned}
E & =-\int_{V \rightarrow \infty} d^{3} x \partial_{j} \Pi^{(0) j} \\
& =-\int_{V \rightarrow \infty} d^{3} x \partial_{j}\left(-4 k e \Sigma^{(0) 0 j}\right) \\
& =-\int_{S \rightarrow \infty} d S_{j}\left(-4 k e \Sigma^{(0) 0 j}\right) \\
& =\int_{r \rightarrow \infty} d \theta d \phi\left(4 k e \Sigma^{(0) 01}\right),
\end{aligned}
$$

The tensor $\Sigma^{(0) 01}=e^{(0)}{ }_{0} \Sigma^{001}+e^{(0)}{ }_{j} \Sigma^{j 01}$ is evaluated by means of Eq. (2). It simplifies to 


$$
\begin{aligned}
\Sigma^{(0) 01}= & \frac{1}{2} e^{(0)}{ }_{0}\left(T^{001}+g^{01} T^{0}\right) \\
& +\frac{1}{2} e^{(0)}{ }_{1}\left(T^{101}+g^{11} T^{0}-g^{01} T^{1}\right) .
\end{aligned}
$$

We need to calculate all components $T_{\lambda \mu \nu}=e^{a}{ }_{\lambda} T_{a \mu \nu}$ of the torsion tensor obtained from Eq. (17). For later purposes, we also provide the asymptotic behavious of these componentes with respect to the radial variable $r$, in the limit $r \rightarrow \infty$. The nonvanishing components are given by

$$
\begin{aligned}
& T_{001}=\frac{1}{2} \partial_{1}\left(A^{2}-C^{2}\right)-A \partial_{0} B \simeq O\left(\frac{1}{r}\right) \\
& T_{101}=B \partial_{1} A \simeq O\left(\frac{1}{r^{2}}\right) \\
& T_{201}=-r e^{\gamma} \partial_{1} C \simeq O\left(\frac{1}{r}\right) \\
& T_{002}=\frac{1}{2} \partial_{2}\left(A^{2}-C^{2}\right)+C\left(\partial_{0} \gamma\right) r e^{\gamma} \simeq O\left(\frac{1}{r}\right) \\
& T_{102}=B \partial_{2} A+B C \simeq O\left(\frac{1}{r}\right) \\
& T_{202}=r e^{\gamma}\left[\left(\partial_{0} \gamma\right) r e^{\gamma}-\partial_{2} C\right] \simeq O(r) \\
& T_{303}=-\left(\partial_{0} \gamma\right) r^{2} e^{-2 \gamma} \sin ^{2} \theta-r e^{-\gamma} C \sin \theta \cos \theta \simeq O(r) \\
& T_{012}=A \partial_{2} B+C\left[e^{\gamma}+\left(\partial_{1} \gamma\right) r e^{\gamma}-B\right] \simeq O\left(\frac{1}{r}\right) \\
& T_{212}=r e^{\gamma}\left[e^{\gamma}+\left(\partial_{1} \gamma\right) r e^{\gamma}-B\right] \simeq O(r) \\
& T_{313}=r e^{-\gamma}\left[e^{-\gamma}-\left(\partial_{1} \gamma\right) r e^{-\gamma}-B\right] \simeq O(r) \\
& T_{323}=-r^{2}\left(\partial_{2} \gamma\right) e^{-2 \gamma} \sin ^{2} \theta-r^{2}\left(1-e^{-2 \gamma}\right) \sin \theta \cos \theta \simeq O\left(r^{2}\right) .
\end{aligned}
$$

We also need the traces $T^{\mu}$. They read

$$
\begin{aligned}
T^{0}= & g^{01} g^{01} T_{101}-g^{01}\left(g^{22} T_{212}+g^{33} T_{313}\right) \\
T^{1}= & -g^{01} g^{01} T_{001}+g^{01} g^{12}\left(T_{012}-T_{201}\right)-g^{11} g^{22} T_{212}-g^{11} g^{33} T_{313} \\
& -g^{01} g^{22} T_{202}-g^{01} g^{33} T_{303}-g^{12} g^{33} T_{323}+g^{12} g^{12} T_{212}
\end{aligned}
$$




$$
\begin{aligned}
& T^{2}=g^{01} g^{12} T_{101}-g^{12} g^{22} T_{212}-g^{12} g^{33} T_{313} \\
& T^{3}=0
\end{aligned}
$$

After the substitution of Eqs. (23) and (24) into Eq. (22) we obtain

$$
\begin{aligned}
\Sigma^{(0) 01}= & -\frac{1}{2} e^{(0)}{ }_{0}\left[g^{01} g^{01} g^{22} T_{212}+g^{01} g^{01} g^{33} T_{313}\right] \\
& +\frac{1}{2} e^{(0)}{ }_{1}\left[g^{01} g^{01} g^{22} T_{202}+g^{01} g^{01} g^{33} T_{303}+g^{01} g^{12} g^{33} T_{323}\right] .
\end{aligned}
$$

With the help of the equation above we arrive at an expression for the total gravitational energy of the space-time,

$$
\begin{aligned}
E= & \frac{1}{4 \pi} \lim _{r \rightarrow \infty} \int_{0}^{2 \pi} d \phi \int_{0}^{\pi} d \theta \frac{1}{2}\left\{r \sin \theta\left[e^{\gamma}+e^{-\gamma}-2 e^{-\beta}\left(\frac{V}{r}\right)^{\frac{1}{2}}\right]\right. \\
& \left.+r^{2} e^{-\beta}\left(\frac{V}{r}\right)^{-\frac{1}{2}} \frac{\partial}{\partial \theta}(U \sin \theta)\right\} \\
= & \frac{1}{4} \lim _{r \rightarrow \infty} \int_{0}^{\pi} d \theta\left\{r \sin \theta\left[e^{\gamma}+e^{-\gamma}-2 e^{-\beta}\left(\frac{V}{r}\right)^{\frac{1}{2}}\right]\right. \\
& \left.+r^{2} e^{-\beta}\left(\frac{V}{r}\right)^{-\frac{1}{2}} \frac{\partial}{\partial \theta}(U \sin \theta)\right\} .
\end{aligned}
$$

Considering the last integral in Eq. (26), we note that

$$
\lim _{r \rightarrow \infty} \int_{0}^{\pi} d \theta r^{2} e^{-\beta}\left(\frac{V}{r}\right)^{-\frac{1}{2}} \frac{\partial}{\partial \theta}(U \sin \theta)=\lim _{r \rightarrow \infty} \int_{0}^{\pi} d \theta r^{2} \frac{\partial}{\partial \theta}(U \sin \theta)
$$

Since the function $U(\theta) \sin \theta$ is required to vanish at $\theta=0, \pi[6]$, we conclude that the integral above vanishes. Moreover we have

$$
e^{\gamma}+e^{-\gamma}-2 e^{-\beta}\left(\frac{V}{r}\right)^{\frac{1}{2}} \simeq \frac{2 M}{r}-\frac{c^{2}}{r^{2}},
$$

in the limit $r \rightarrow \infty$. Therefore in such limit we obtain

$$
E=\frac{1}{2} \int_{0}^{\pi} d \theta \sin \theta M(u, \theta)
$$


which is precisely the expression of the Bondi energy.

It is possible to show by explicit calculations that the Bondi energy is invariant under local Lorentz transformations, $\tilde{e}^{a}{ }_{\mu}(x)=\Lambda^{a}{ }_{b}(x) e^{b}{ }_{\mu}(x)$, whose asymptotic behaviour is given by

$$
\begin{aligned}
& \Lambda^{(0)}{ }_{(0)}=1+{ }^{1} \omega^{(0)}{ }_{(0)}(1 / r) \\
& \Lambda^{(0)}{ }_{(i)}={ }^{1} \omega^{(0)}{ }_{(i)}(1 / r) \\
& \Lambda^{(i)}{ }_{(j)}=\delta_{(j)}^{(i)}+{ }^{0} \omega^{(i)}{ }_{(j)}+{ }^{1} \omega^{(i)}{ }_{(j)}(1 / r),
\end{aligned}
$$

where ${ }^{0} \omega_{(i)(j)}$ and ${ }^{1} \omega_{a b}$ are infinitesimal quantities such that ${ }^{0} \omega_{(i)(j)}=$ $-{ }^{0} \omega_{(i)(j)},{ }^{1} \omega_{a b}=-{ }^{1} \omega_{b a}$, and ${ }^{0} \omega_{(i)(j)}$ are constants. This result is no surprise since Eq. (7) can be evaluated as a surface integral, and all transformations that preserve the asymptotic structure of the field quantities are allowed. Nevertheless it is possible to show by explicit calculations that in the limit $r \rightarrow \infty$ we have

$$
\begin{aligned}
& \tilde{T}_{212}=T_{212} \\
& \tilde{T}_{313}=T_{313} \\
& \tilde{T}_{202}=T_{202} \\
& \tilde{T}_{303}=T_{303} \\
& \tilde{T}_{323}=T_{323},
\end{aligned}
$$

and therefore

$$
\begin{aligned}
\tilde{\Sigma}^{(0) 01}= & -\frac{1}{2}\left(\Lambda^{(0)}{ }_{(0)} e^{(0)}{ }_{0}+\Lambda_{(i)}^{(0)} e^{(i)}{ }_{0}\right)\left[g^{01} g^{01} g^{22} T_{212}+g^{01} g^{01} g^{33} T_{313}\right] \\
& +\frac{1}{2}\left(\Lambda^{(0)}{ }_{(0)} e^{(0)}{ }_{1}+\Lambda^{(0)}{ }_{(i)} e^{(i)}{ }_{1}\right)\left[g^{01} g^{01} g^{22} T_{202}+g^{01} g^{01} g^{33} T_{303}\right. \\
& \left.+g^{01} g^{12} g^{33} T_{323}\right]
\end{aligned}
$$

from what we conclude that Eq. (21) is invariant under the transformations given by Eq. (30). We note that in the expression above the tetrad components $e^{(i)}{ }_{1}$ behave as $r^{0}$ when $r \rightarrow \infty$. 
The flux of gravitational energy is determined by the $a=(0)$ component of Eq. (9), and by making $j=1$, i.e., by integrating over a surface $S$ of constant radius $r$, and requiring $r \rightarrow \infty$. It reads

$$
\begin{aligned}
\Phi_{g}^{(0)}= & k \int_{S} d S_{j}\left[e e^{(0) \mu}\left(4 \Sigma^{b c j} T_{b c \mu}-\delta_{\mu}^{j} \Sigma^{b c d} T_{b c d}\right)\right] \\
= & k \int_{S} d S_{1} e\left[g^{01} e^{(0)}{ }_{0} \Omega^{1}{ }_{1}+g^{01} e^{(0)}{ }_{1} \Omega^{1}{ }_{0}\right. \\
& \left.+g^{11} e^{(0)}{ }_{1} \Omega^{1}{ }_{1}+g^{12} e^{(0)}{ }_{1} \Omega^{1}{ }_{2}\right]
\end{aligned}
$$

where

$$
\begin{aligned}
& \Omega^{1}{ }_{1}=4 \Sigma^{\lambda \sigma 1} T_{\lambda \sigma 1}-\Sigma^{\lambda \sigma \nu} T_{\lambda \sigma \nu}, \\
& \Omega^{1}{ }_{0}=4 \Sigma^{\lambda \sigma 1} T_{\lambda \sigma 0} \text {, } \\
& \Omega^{1}{ }_{2}=4 \Sigma^{\lambda \sigma 1} T_{\lambda \sigma 2} \text {. }
\end{aligned}
$$

The expression of the quantities above are given in the Appendix. In order to calculate the total flux in the limit $r \rightarrow \infty$ we take into account the asymptotic behaviour of the following field quantities,

$$
\begin{aligned}
g^{01} & \rightarrow-1, \\
g^{11} & \rightarrow 1, \\
g^{12} & \rightarrow O\left(1 / r^{2}\right), \\
e^{(0)} & \rightarrow 1, \\
e^{(0)}{ }_{0} & \rightarrow 1, \\
e & \rightarrow r^{2} \sin \theta .
\end{aligned}
$$

It is easy to verify that in the limit $r \rightarrow \infty$ the first and third term on the right hand side of Eq. (33) cancel each other, and that the last term behaves as $g^{12} e^{(0)}{ }_{1} \Omega^{1}{ }_{2}=O\left(1 / r^{4}\right)$. Therefore $\Phi_{g}^{(0)}$ reduces to

$$
\Phi_{g}^{(0)}=\frac{1}{16 \pi} \int_{S \rightarrow \infty} d \theta d \phi e\left(-\Omega^{1}{ }_{0}\right) .
$$

In order to simplify the evaluation of the integral above we only consider terms in Eq. (A.2) that fall off as $1 / r^{2}$. For this purpose we take into account 
Eqs. (23) and (35). We conclude that five terms may possibly contribute to the surface integral when $r \rightarrow \infty$. By integrating in $\phi$ we find that in this limit Eq. (36) simplifies to

$$
\begin{aligned}
\Phi_{g}^{(0)}= & \frac{1}{4} \int_{S \rightarrow \infty} d \theta\left(r^{2} \sin \theta\right)\left[g^{01} g^{01} g^{22} T_{001} T_{202}+g^{01} g^{01} g^{33} T_{001} T_{303}\right. \\
& \left.g^{11} g^{22} g^{33}\left(T_{202} T_{313}+T_{303} T_{212}\right)+2 g^{01} g^{22} g^{33} T_{202} T_{303}\right] .
\end{aligned}
$$

By explicitly calculating the asymptotic behaviour of all quantities under integration, and after carrying out several cancellations, we conclude that only one term arising from the product $T_{202} T_{303}$ in Eq. (37) yields a nonvanishing value to $\Phi^{(0)}$. We obtain

$$
\Phi_{g}^{(0)}=\frac{1}{2} \int_{0}^{\pi} d \theta \sin \theta\left(\partial_{0} c\right)^{2}
$$

which is the well known value of the loss of mass in Bondi's space-time [6]. We note that no multiplicative factors had to be adjusted in Eqs. (9) or (33), in order to arrive at the expression above. To our knowledge it is the first time that the loss of mass has been obtained by means of a flux equation for the gravitational energy. Finally we note that since Eq.(14) is a vacuum solution of Einstein's equations, then $\Phi_{m}^{(0)}=0$ and thus Eq. (11) is naturally verified.

\section{The Vaidya metric tensor}

The Vaidya metric [15] describes the exterior geometry of a spherically symmetric star when the energy flux of matter is included. The star is considered to be throwing away mass. A spherically symmetric matter distribution does not emit gravitational radiation by means of gravitational waves, as in Bondi's space-time. Therefore in the present context the energy flux is a purely matter flux. The metric is determined by the mass function $m(u)$, which is a nonincreasing function of the retarded time. It reads

$$
d s^{2}=-\left(1-\frac{2 m(u)}{r}\right) d u^{2}-2 d u d r+r^{2}\left(d \theta^{2}+\sin \theta^{2} d \phi^{2}\right) .
$$


If we require the function $m$ to be constant, then the metric tensor above reduces to the Schwarzschild solution.

The set of tetrad fields that yields Eq. (39) is obtained by following the same steps that led to Eq. (17). We require $e^{a}{ }_{\mu}$ to satisfy a condition similar to Eq. (19), i.e., the isotropic violation of the time gauge condition, together with Eq. (20). The tetrad field for which both conditions are satisfied is given by

$$
e_{a \mu}(u, r, \theta, \phi)=\left(\begin{array}{cccc}
-\alpha & -\alpha^{-1} & 0 & 0 \\
0 & \alpha^{-1} \sin \theta \cos \phi & r \cos \theta \cos \phi & -r \sin \theta \sin \phi \\
0 & \alpha^{-1} \sin \theta \sin \phi & r \cos \theta \sin \phi & r \sin \theta \cos \phi \\
0 & \alpha^{-1} \cos \theta & -r \sin \theta & 0
\end{array}\right)
$$

where $\alpha=(1-2 m / r)^{1 / 2}$. The nonvanishing components of $T_{\mu \nu \lambda}=e^{a}{ }_{\mu} T_{a \nu \lambda}$ are

$$
\begin{aligned}
T_{001} & =-\frac{\dot{m}}{\alpha^{2} r}+\frac{m}{r^{2}}, \\
T_{101} & =\frac{m}{\alpha^{2} r^{2}}, \\
T_{212} & =r\left(1-\alpha^{-1}\right), \\
T_{313} & =r\left(1-\alpha^{-1}\right) \sin \theta^{2},
\end{aligned}
$$

with $\dot{m}=d m / d u$. The calculations in this context are much simpler than in the preceeding case. We have

$$
\begin{aligned}
\Sigma^{(0) 01}= & e^{(0)}{ }_{0} \Sigma^{001}+e^{(0)}{ }_{1} \Sigma^{101} \\
= & \frac{1}{2} e^{(0)}{ }_{0}\left(T^{001}+g^{01} T^{0}\right) \\
& +\frac{1}{2} e^{(0)}{ }_{1}\left(T^{101}+g^{11} T^{0}-g^{01} T^{1}\right) .
\end{aligned}
$$

After a number of simplifications we obtain

$$
\begin{aligned}
\Sigma^{(0) 01} & =-\frac{1}{2} e^{(0)}{ }_{0}\left(g^{22} T_{212}+g^{33} T_{313}\right) \\
& =\frac{1}{r}(1-\alpha) .
\end{aligned}
$$


Therefore by considering a finite volume $V$, enclosed by a spherical surface $S$ of radius $r$, we have

$$
\begin{aligned}
E(u) & =-\int_{V} d^{3} x \partial_{j} \Pi^{(0) j} \\
& =\int_{S} d \theta d \phi\left(4 k e \Sigma^{(0) 01}\right) \\
& =r\left(1-\sqrt{1-\frac{2 m(u)}{r}}\right) .
\end{aligned}
$$

The total gravitational energy of the space-time is obtained by making $r \rightarrow$ $\infty$. It is given by $E_{\text {total }}=m(u)$. From Eq. (44) it follows that

$$
\frac{d E(u)}{d u}=\frac{\dot{m}(u)}{\sqrt{1-\frac{2 m(u)}{r}}} .
$$

Now we proceed to evaluate the fluxes $\Phi_{g}^{(0)}$ and $\Phi_{m}^{(0)}$. The former is given by

$$
\begin{aligned}
\Phi_{g}^{(0)} & =k \int_{S} d S_{j}\left[e e^{(0) \mu}\left(4 \Sigma^{b c j} T_{b c \mu}-\delta_{\mu}^{j} \Sigma^{b c d} T_{b c d}\right)\right] \\
& =k \int_{S} d S_{1} e\left[g^{01} e^{(0)}{ }_{0} \Omega^{1}{ }_{1}+g^{01} e^{(0)}{ }_{1} \Omega^{1}{ }_{0}+g^{11} e^{(0)}{ }_{1} \Omega^{1}{ }_{1}\right]
\end{aligned}
$$

where $S$ is a surface of constant radius $r$ and the quantities $\Omega_{\nu}^{\mu}$ are defined by Eq. (34). For the Vaidya metric tensor we have $g^{00}=0, g^{01}=-1, g^{11}=\alpha^{2}$ and $g^{12}=0$. By taking into account the nonvanishing components of $T_{\mu \nu \lambda}$ it is possible to conclude that in the present case we find

$$
\begin{aligned}
& \Omega^{1}{ }_{0}=0 \\
& \Omega_{1}^{1}=\Sigma^{\mu \nu \lambda} T_{\mu \nu \lambda} .
\end{aligned}
$$

Moreover we have $g^{01} e^{(0)}{ }_{0}=-\alpha$ and $g^{11} e^{(0)}{ }_{1}=\alpha$, from what we conclude that $\Phi_{g}^{(0)}=0$, as expected.

In order to calculate the energy flux of matter $\Phi_{m}^{(0)}$ we need the energymomentum tensor associated to Eq. (39). It reads [16] 


$$
T_{\mu \nu}=-\frac{1}{4 \pi r^{2}} \frac{d m(u)}{d u} \delta_{\mu}^{0} \delta_{\nu}^{0}
$$

For a volume $V$ enclosed by a surface of constant radius $S$ we find

$$
\begin{aligned}
\Phi_{m}^{(0)} & =\int_{V} d^{3} x \partial_{j}\left(e e^{(0)}{ }_{\mu} T^{j \mu}\right) \\
& =\int_{S} d S_{1} e e^{(0)}{ }_{\mu} T^{1 \mu} \\
& =\int_{S} d \theta d \phi\left(r^{2} \sin \theta\right) e^{(0)}{ }_{1} T^{11} .
\end{aligned}
$$

Given that $T^{11}=-\dot{m} /\left(4 \pi r^{2}\right)$, we obtain

$$
\Phi_{m}^{(0)}=-\frac{\dot{m}}{\sqrt{1-\frac{2 m(u)}{r}}},
$$

Therefore Eqs. (45) and (50) are in agreement with Eq. (11). We conclude that in the Vaidya space-time the energy flux of matter accounts for the loss of gravitational energy. By taking the limit $r \rightarrow \infty$ in Eqs. (45) and (50) we recover the well known result of the total flux (total luminosity $L_{\infty}$ ) measured by an observer at infinity, $L_{\infty}=-d m / d u[16]$.

\section{Concluding remarks}

The flux of the energy-momentum of gravitational and matter fields has not been investigated so far in the literature in a unique geometrical setting. In particular, to our knowledge the flux of matter fields $\Phi_{m}^{a}$ defined by Eq. (10) has not been previously considered. The total flux of matter fields does not depend on the particular structure of the tetrad fields, but only on the asymptotic behaviour of the latter. The remarkable feature of the continuity equation (8) is that it is a natural consequence of Einstein's equations written in the TEGR. The detailed analysis of Eq. (11) in the context of Bondi and Vaidya space-times demonstrates the consistency of the present framework for investigating the energy-momentum properties of the gravitational field. One may then consider the application of the techniques presented here to 
concrete astrophysical problems, in which case one may assume the surface $S$ in Eq. (9) to be an open surface, as considered in Ref. [11]. In the latter reference we evaluated the energy flux of plane gravitational waves. It is likely that for a given set of tetrad fields a nonvanishing value of $\Phi_{g}^{(0)}$ indicates the emission of gravitational waves. However, Bondi argues that the gravitational energy can be transferred by means of two distinct ways, radiative transfer and inductive transfer [12]. Therefore it is possible that a nonvanishing value of $\Phi_{g}^{(0)}$ also indicates a form of inductive transfer of gravitational energy. This issue will be further analyzed.

Restricting considerations to spherical surfaces $S$ of constant radius, it is possible to show by explicit calculations that for the Bondi space-time the three momenta $P^{(i)}$ given by Eq. (7) vanish. However, we note that $P^{(3)}$ vanishes only by placing the surface $S$ at spacelike infinity. For a spherical surface $S$ of finite radius $r, P^{(3)}$ is nonvanishing. The physical meaning of a nonvanishing $P^{(i)}$ is under investigation.

It is an interesting achievement of the present approach to the energymomentum of the gravitational field that the definition given by Eq. (7) allows a unfied treatment of the Bondi and ADM energies. For this purpose we have to fix suitable expressions for the tetrad field in each case. Although the localization of the gravitational energy depends on the choice of the tetrad field, total quantities are in fact invariant under a wide class of local Lorentz transformations.

\section{Acknowledgements}

F. F. Faria is grateful to the Brazilian agency CAPES for financial support.

\section{APPENDIX}

For the Bondi space-time metric tensor the quantities $\Omega^{\mu}{ }_{\nu}$ are given by

$$
\begin{gathered}
\Omega_{1}^{1}=g^{01} g^{01} g^{22}\left(\frac{1}{2} T_{012} T_{012}+T_{012} T_{201}-\frac{1}{2} T_{201} T_{201}\right. \\
\left.-2 T_{001} T_{212}-\frac{1}{2} T_{102} T_{102}\right) \\
-2 g^{01} g^{01} g^{33} T_{001} T_{313}+2 g^{01} g^{12} g^{22} T_{012} T_{212}
\end{gathered}
$$




$$
\begin{gathered}
+2 g^{01} g^{12} g^{33}\left(T_{012} T_{313}-T_{201} T_{313}\right) \\
+g^{12} g^{12} g^{22} T_{212} T_{212}+2 g^{12} g^{12} g^{33} T_{212} T_{313} \\
-2 g^{11} g^{22} g^{33} T_{212} T_{313}-g^{22} g^{33} g^{33} T_{323} T_{323} \\
\Omega_{0}^{1}=g^{01} g^{01} g^{22}\left(3 T_{002} T_{102}+T_{002} T_{012}+T_{002} T_{201}\right. \\
\left.-2 T_{202} T_{001}\right)-2 g^{01} g^{01} g^{33} T_{001} T_{303}+2 g^{11} g^{12} g^{22} T_{102} T_{212} \\
+2 g^{01} g^{12} g^{22}\left(T_{002} T_{212}+T_{202} T_{102}+T_{202} T_{012}\right) \\
+2 g^{01} g^{12} g^{33}\left(T_{002} T_{313}-T_{102} T_{303}+T_{012} T_{303}\right. \\
+2 g^{01} g^{22} g^{33} T_{202} T_{323}-2 g^{11} g^{22} g^{33} T_{212} T_{323}+2 g^{12} g^{12} g^{33} T_{212} T_{323} \\
\left.T_{303} T_{201}\right)+2 g^{01} g^{11} g^{22}\left(T_{102} T_{012}+T_{102} T_{102}\right) \\
+2 g^{12} g^{12} g^{33}\left(-T_{102} T_{323}+T_{202} T_{313}+T_{303} T_{212}\right) \\
-2 g^{11} g^{22} g^{33}\left(T_{202} T_{313}+T_{212} T_{303}\right)-4 g_{002} T_{212}-T_{201} T_{202}+T_{202} T_{102} \\
+2 g^{01} g^{01} g^{33}\left(T_{102} T_{303}-T_{001} T_{323}-T_{002} T_{313}\right)
\end{gathered}
$$




\section{References}

[1] L. D. Landau and E. M. Lifshitz, The Classical Theory of Fields (Pergamon Press, Oxford, 1980).

[2] S. Weinberg, Gravitation and Cosmology: Principles and Applications of the General Theory of Relativity (Wiley, New York, 1972).

[3] J. N. Goldberg, "Invariant Transformations, Conservation Laws and Energy-Momentum", in General Relativity and Gravitation: One Hundred Years After the Birth of Albert Einstein, edited by A. Held, Vol. 1, p. 469 (Plenum, New York, 1980).

[4] C.-M. Chen and J. M. Nester, Gravitation and Cosmology 6, 257 (2000); C. C. Chang, J. M. Nester and C.-M. Chen, Phys. Rev. Lett. 83, 1897 (1999).

[5] R. Arnowitt, S. Deser and C. W. Misner, in Gravitation: an Introduction to Current Research, edited by L. Witten (Wiley, New York, 1962).

[6] H. Bondi, M. G. J. van der Burg, and A. W. K. Metzner, Proc. R. Soc. London, Ser. A 269, 21 (1962).

[7] J. W. Maluf, J. F. da Rocha-Neto, T. M. L. Toríbio and K. H. CastelloBranco, Phys. Rev. D 65, 124001 (2002).

[8] J. W. Maluf, J. Math. Phys. 36, 4242 (1995).

[9] F. W. Hehl, J. D. McCrea, E. W. Mielke and Y. Ne'eman, Phys. Rep. 258, 1 (1995).

[10] C. Møller, "Tetrad Fields and Conservation Laws in General Relativity", Proceedings of the International School of Physics Enrico Fermi, edited by C. Møller (Academic Press, London, 1962); "Conservation Laws in the Tetrad Theory of Gravitation", Proceedings of the Conference on Theory of Gravitation, Warszawa and Jablonna 1962 (GauthierVillars, Paris, and PWN-Polish Scientific Publishers, Warszawa, 1964) (NORDITA Publications No. 136).

[11] J. W. Maluf, F. F. Faria and K. H. Castello-Branco, Class. Quantum Grav. 20, 4683 (2003). 
[12] H. Bondi, Proc. R. Soc. Lond. A 427, 249 (1990).

[13] J. L. Synge, Relativity: the General Theory (North Holland, Amsterdam, 1960).

[14] R. Aldrovandi, P. B. Barros and J. G. Pereira, Found. Phys. 33, 545 (2003).

[15] P. C. Vaidya, Nature 171, 260 (1953).

[16] R. W. Lindquist, R. A. Schwartz and C. W. Misner, Phys. Rev. 137, B1364 (1965).

[17] J. W. Maluf, J. Math. Phys. 35. 335 (1994).

[18] J. W. Maluf and J. F. da Rocha-Neto, Phys. Rev. D 64, 084014 (2001).

[19] M. Blagojević and I. A. Nikolić, Phys. Rev. D 62, 024021 (2000).

[20] J. W. Maluf and J. F. da Rocha-Neto, Gen. Rel. Grav. 31, 173 (1999).

[21] J. W. Maluf and J. F. da Rocha-Neto, J. Math. Phys. 40, 1490 (1999).

[22] A. Albrecht and J. Magueijo, Phys. Rev. D 59, 043516 (1999); J. Magueijo, Rept. Prog. Phys. 66, 2025 (2003); M. Arminjon, "Ether theory of gravitation: why and how?" [gr-qc/0401021]; M. Arminjon, "Gravitational effects on light rays and binary pulsar energy loss in a scalar theory of gravity", Theor. Math. Phys. (Teor. Mat. Fiz.), to appear [gr-qc/0301062]; M. Arminjon, "Point-particle limit in a scalar theory of gravitation and the weak equivalence principle" [gr-qc/0306025]; M. Consoli and E. Costanzo, "The motion of the solar system and the Michelson-Morley experiment" [astro-ph/0311576]. 